\title{
THE STRUCTURE OF DISCONTINUOUS HOMOMORPHISMS FROM NON-COMMUTATIVE C*-ALGEBRAS
}

\author{
by VOLKER RUNDE
}

(Received 30 October, 1992)

Introduction. Let $X$ be a compact Hausdorff space, let $C(X)$ denote the algebra of all continuous functions on $X$, let $B$ be a Banach algebra, and let $\theta: C(X) \rightarrow B$ be a (possibly discontinuous) homomorphism with dense range. A classical theorem by W. G. Bade and P. C. Curtis ([2, Theorem 4.3]) describes in great detai] the structure of $\theta$; we shall refer to this result as the Bade-Curtis theorem. Before we give a brief sketch of this theorem, we fix some notation. For $Y \subset X$ let $I(Y)$ and $J(Y)$ denote the ideals of all functions in $C(X)$ that vanish on $Y$ and on a neighborhood of $Y$ respectively; if $Y=\{x\}$ for some $x \in X$, we write $m_{x}$ and $J_{x}$ for $I(Y)$ and $J(Y)$ respectively. According to the Bade-Curtis theorem there is a finite set $\left\{x_{1}, \ldots, x_{n}\right\} \subset X$, the so-called singularity set of $\theta$, such that $\theta \mid J\left(\left\{x_{1}, \ldots, x_{n}\right\}\right)$ is continuous. As a consequence, the restriction of $\theta$ to the dense subalgebra of $C(X)$ consisting of all those functions which are constant near each $x_{j}(j=1, \ldots, n)$ is continuous, and extends to a continuous homomorphism $\theta_{\text {cont }}: C(X) \rightarrow B$. Let $\theta_{\text {sing }}:=\theta-\theta_{\text {cont }}$. Then $\theta_{\text {sing }} \mid I\left(\left\{x_{1}, \ldots, x_{n}\right\}\right)$ is a homomorphism onto a dense subalgebra of $\operatorname{rad}(B) . \theta_{\text {cont }}$ and $\theta_{\text {sing }}$ are called the continuous and the singular part of $\theta$ respectively. Moreover, there are linear maps $\theta_{\text {sing }}^{(1)}, \ldots, \theta_{\text {sing }}^{(\prime)}: C(X) \rightarrow$ $B$ such that

(i) $\theta_{\text {sing }}=\theta_{\text {sing }}^{(1)}+\ldots+\theta_{\text {sing }}^{(n)}$,

(ii) $\theta_{\text {sing }}^{(j)} \mid \mathfrak{m}_{x_{i}}(j=1, \ldots, n)$ is a homomorphism, and

(iii) $\theta_{\text {sing }}^{(j)}\left(J_{x_{i}}\right)=\{0\}(j=1, \ldots, n)$.

Condition (iii) forces the homomorphisms $\theta_{\text {sing }}^{(1)}\left|\mathfrak{m}_{x_{1}}, \ldots, \theta_{\text {sing }}^{(n)}\right| \|_{x_{n}}$ to map into $\operatorname{rad}(B)$; such homomorphisms are called radical homomorphisms.

When H. G. Dales and J. Esterle independently proved-assuming the continuum hypothesis-that if $X$ is infinite, then there is a discontinuous homomorphism from $C(X)$ into a Banach algebra $([3],[6],[8]$ and [7]), they did so by constructing a radical homomorphism from a maximal ideal of $C(X)$.

At least parts of the Bade-Curtis theorem on the structure of a discontinuous homomorphism from $C(X)$ still hold in the non-commutative case; i.e. with $C(X)$ replaced by an arbitrary $C^{*}$-algebra. See ([20, Theorem 4.1], [23], and [13, Corollary 8]). Let $A$ be a $C^{*}$-algebra, let $B$ be a Banach algebra, and let $\theta: A \rightarrow B$ be a homomorphism with dense range; let $\mathscr{I}(\theta)$ denote the continuity ideal of $\theta$. Then $\overline{\mathscr{I}(\theta)}$ has finite codimension in $A$, and there is a dense subalgebra of $A$ containing $\mathscr{I}(\theta)$ such that the restriction of $\theta$ to this subalgebra is continuous. Let $\theta_{\text {cont }}: A \rightarrow B$ be the continuous extension of this restriction, and put $\theta_{\text {sing }}:=\theta-\theta_{\text {cont }}$. Then $\theta_{\text {sing }} \mid \overline{\mathscr{I}(\theta)}$ is a homomorphism onto a dense subalgebra of $\Xi(\theta)$, where $\Xi(\theta)$ is the separating space of $\theta$, which contains $\operatorname{rad}(B)$. Comparing the Bade-Curtis theorem and its partial non-commutative analogue, two questions arise naturally.

1. Does $\theta_{\text {sing }} \operatorname{map} \overline{\mathscr{I}(\theta)}$ into $\operatorname{rad}(B)$ ? (This is equivalent to the big open problem of whether every homomorphism from a $\mathrm{C}^{*}$-algebra onto a dense subalgebra of a semisimple Banach algebra is automatically continuous.)

2. Does a further splitting of $\theta_{\text {sing }}$ as in the commutative case exist in general?

Glasgow Math. J. 36 (1994) 209-218. 
In the present paper, we investigate these questions. We proceed as follows. In the first section, we put together some preliminary material for easier reference later.

Then, in Section 2, we show that under certain conditions the singular part of a homomorphism from a $\mathrm{C}^{*}$-algebra $A$ admits a splitting as in the commutative case (Theorem 2.3); these conditions are automatically satisfied whenever $A$ is an $\mathrm{AW}^{*}$ algebra or a unital $C^{*}$-algebra whose primitive spectrum $\operatorname{Prim}(A)$ is Hausdorff. In these two cases, we also have that $\theta_{\text {sing }} \operatorname{maps} \overline{\mathscr{I}(\theta)}$ into $\operatorname{rad}(B)($ Corollary 2.4$)$.

As we shall see in the third section, not all assertions of the Bade-Curtis theorem carry over to the non-commutative situation. We give an example of a homomorphism from a separable liminal $C^{*}$-algebra for whose singular part no further splitting exists.

Finally, in section four, we extend some results by $B$. E. Johnson $([\mathbf{1 0}])$ on the structure of discontinuous homomorphisms from $C(X)$ to the non-commutative setting (Theorem 4.2 and Theorem 4.3).

1. Preliminaries. We begin with the notion of the separating space of a linear operator, a concept which is fundamental in automatic continuity.

Definition 1.1. Let $E$ and $F$ be Banach spaces, and let $\theta: E \rightarrow F$ be a linear operator. Then

$\Xi(\theta):=\left\{y \in F:\right.$ there is a sequence $\left\{x_{n}\right\}_{n=1}^{x}$ in $E$ such that $x_{n} \rightarrow 0$ and $\left.\theta\left(x_{n}\right) \rightarrow y\right\}$

is called the separating space of $\theta$.

It is easy to see that $\Xi(\theta)$ is a closed, linear subspace of $F$. In terms of Definition 1.1, the closed graph theorem states that $\theta$ is continuous if and only if $\Xi(\theta)=\{0\}$. For more information about separating spaces see the first chapter of A. M. Sinclair's monograph [22].

If $A$ and $B$ are Banach algebras, and $\theta: A \rightarrow B$ is a homomorphism, then $\Xi(\theta)$ is a closed ideal of $\overline{\theta(A)}$. In this context another ideal is of importance for the continuity properties of $\theta$.

Definition 1.2. Let $A$ and $B$ be Banach algebras, and let $\theta: A \rightarrow B$ be a homomorphism. Then

$$
\mathscr{I}(\theta):=\{a \in A: \theta(a) \Xi(\theta)=\Xi(\theta) \theta(a)=\{0\}\}
$$

is called the continuity ideal of $\theta$.

The concept of the continuity ideal of a homomorphism was introduced by B. E. Johnson in [9] with a slightly different definition that lacked symmetry. The present definition is due to A. M. Sinclair ([20]).

The following proposition collects the essential facts about the continuity ideal when the domain is a $C^{*}$-algebra.

Proposition 1.3. Let $A$ be a $C^{*}$-algebra and let $\theta: A \rightarrow B$ be a homomorphism into a Banach algebra $B$. Then

(i) $\mathscr{I}(\theta)$ is an ideal whose closure has finite codimension,

(ii) $\theta$ is continuous if and only if $\mathscr{I}(\theta)$ is closed,

(iii) if $I \subset A$ is an ideal, then $\theta \mid I$ is continuous if and only if $I \subset \mathscr{I}(\theta)$.

Proof. (i) is part of [20, Theorem 3.8], (ii) is [22, Remark 12.3], and (iii) is stated as [11, Remark 1]. 
Let $X$ be a compact Hausdorff space, let $B$ be a Banach algebra, and let $\theta: C(X) \rightarrow B$ be a homomorphism onto a dense subalgebra of $B$ with singularity set $\left\{x_{1}, \ldots, x_{n}\right\}$. The fact that

$$
J\left(\left\{x_{1}, \ldots, x_{n}\right\}\right) \subset \mathscr{I}(\theta) \subset \overline{\mathscr{I}(\theta)}=I\left(\left\{x_{1}, \ldots, x_{n}\right\}\right)
$$

plays a crucial role in the proof of the commutative splitting result. To obtain a non-commutative analogue, we need a substitute for $J\left(\left\{x_{1}, \ldots, x_{n}\right\}\right)$ in the context of arbitrary $\mathrm{C}^{*}$-algebras. An appropriate replacement was introduced by $\mathrm{G}$. K. Pedersen in [15]; its usefulness in the study of discontinuous homomorphisms was first observed by K. B. Laursen and A. M. Sinclair ([13, Corollary 8]).

For a $C^{*}$-algebra $A$ let $A^{+}$denote the cone of its positive elements.

Definition 1.4. Let $A$ be a $C^{*}$-algebra. Put

$$
\mathscr{P}_{0}(A):=\left\{a \in A^{+}: \text {there is } x \in A^{+} \text {such that } a x=a\right\}
$$

let

$$
\mathscr{P}^{+}(A):=\left\{a \in A^{+}: \text {there are } x_{1}, \ldots, x_{n} \in \mathscr{P}_{0}(A) \text { such that } a \leq x_{1}+\ldots+x_{n}\right\},
$$

and define $\mathscr{P}(A)$ as the linear span of $\mathscr{P}^{+}(A)$. Then $\mathscr{P}(A)$ is called the Pedersen ideal of A.

If $A=C_{0}(X)$ for a locally compact Hausdorff space $X$, then $\mathscr{P}(A)$ is the ideal $C_{(\kappa)}(X)$ of all continuous functions on $X$ having compact support.

TheOREM 1.5. Let $A$ be a $C^{*}$-algebra. Then $\mathscr{P}(A)$ is a dense hereditary ideal of $A$, and equals the intersection of all dense ideals of $A$.

Proof. Although our definition of $\mathscr{P}(A)$ apparently defines a larger set than the definition in [17] does, an inspection of the proof of $[17,5.6 .1]$ reveals that the definitions are equivalent (see also [14, Proposition 2.3]).

If $X$ is a locally compact Hausdorff space, and $\theta: C_{0}(X) \rightarrow B$ a homomorphism, where $B$ is a commutative Banach algebra, then $\theta \operatorname{maps}$ into $\operatorname{rad}(B)$ if and only if $\theta\left(C_{00}(X)\right)=\{0\}$. This statement has a non-commutative analogue.

For a Banach algebra $B$, let $\mathscr{2}(B)$ denote the set of its quasinilpotent elements.

Proposition 1.6. Let $A$ be a $C^{*}$-algebra, let $B$ be a Banach algebra and let $\theta: A \rightarrow B$ be a homomorphism. Then $\theta(\mathscr{P}(A))=\{0\}$ if and only if $\theta(A) \subset \mathscr{2}(B)$.

Proof. Assume that $\theta(\mathscr{P}(A))=\{0\}$. We have an isomorphism of complex algebras $A / \operatorname{ker} \theta \cong \theta(A)$. Since $\mathscr{P}(A)$ and hence $\operatorname{ker} \theta$ is dense in $A, A / \operatorname{ker} \theta$ is a radical algebra; (see, for example, [13, Lemma 4]). Hence $\theta(A)$ is a normed radical algebra; i.e. for each $x \in \theta(A)$ we have $\sigma_{B}(x) \subset \sigma_{\theta(A)}(x)=\{0\}$.

Conversely assume $\theta(A) \subset \mathscr{Q}(B)$. First, let $a \in \mathscr{P}_{0}(A)$. Suppose that there is $x \in A$ such that $a x=a$. Inductively, we obtain $a x^{\prime \prime}=a(n \in \mathbb{N})$, and therefore

$$
\|\theta(a)\|^{1 / n} \leq\|\theta(a)\|^{1 / n}\left\|\theta(x)^{n}\right\|^{1 / n} \quad(n \in \mathbb{N}) .
$$

Since $\theta(x) \in \mathscr{Q}(B), \lim _{n \rightarrow x}\|\theta(a)\|^{1 / n}=0$, which is possible only if $\theta(a)=0$. Now, let $a \in \mathscr{P}^{+}(A)$. By definition, there are $x_{1}, \ldots, x_{n} \in \mathscr{P}_{0}(A)$ such that $a \leq x_{1}+\ldots+x_{n}$. Since $x_{1}+\ldots+x_{n}$ is self-adjoint and $\theta\left(x_{1}+\ldots+x_{n}\right)=0$ by the foregoing, we have by 
[21, Lemma 4.1] that $\theta\left(\left(x_{1}+\ldots+x_{n}\right)^{1 / 2}\right)=0$. The generalized polar decomposition $([17,1.4 .5])$ yields $u \in A$ such that $a=u\left(x_{1}+\ldots+x_{n}\right)^{1 / 2}$, where $\theta(a)=0$. It is clear now that $\theta(\mathscr{P}(A))=\{0\}$.

Remark. To show that $\theta(\mathscr{P}(A))=\{0\}$ it actually suffices to assume that $\theta\left(A^{+}\right) \subset$ $2(B)$. Hence, we have

$$
\theta(A) \subset 2(B) \Leftrightarrow \theta\left(A^{+}\right) \subset 2(B) .
$$

2. A non-commutative splitting result. Our goal in this section is to establish a splitting result for the singular part of discontinuous homomorphisms from certain non-commutative $\mathrm{C}^{*}$-algebras.

To make formulations less cumbersome, we introduce a definition.

Definition 2.1. Let $A$ be a unital $C^{*}$-algebra, and let $\theta: A \rightarrow B$ be a homomorphism into a Banach algebra $B$. We say that $\theta$ admits a splitting if there are linear maps $\theta_{\text {sing }}^{(1)}, \ldots, \theta_{\text {sing }}^{(n)}: A \rightarrow B$ such that the following conditions hold.

(i) $\theta_{\text {sing }}=\theta_{\text {sing }}^{(1)}+\ldots+\theta_{\text {sing }}^{(n)}$.

(ii) $\Xi(\theta)=\overline{\theta_{\text {sing }}^{(1)}(A)} \oplus \ldots \oplus \overline{\theta_{\text {sing }}^{(1)}(A)}$.

(iii) $\theta_{\text {sing }}^{(j)}(A) . \theta_{\text {sing }}^{(k)}(A)=\{0\} \quad(j \neq k)$.

(iv) Let $m_{1}, \ldots, m_{n}$ be the maximal ideals in hull $(\overline{\mathscr{F}(\theta)})$. Then $\theta_{\text {sing }}^{(j)} \mid m_{j}$ is a homomorphism for $j=1, \ldots, n$.

(v) $\theta_{\text {sing }}^{(j)}\left(\mathscr{P}\left(\mathrm{m}_{j}\right)\right)=\{0\} \quad(j=1, \ldots, n)$.

The part of the Bade-Curtis theorem we want to generalize then plainly states that every homomorphism from $C(X)$, where $X$ is a compact Hausdorff space, admits a splitting, and the problem we are concerned with reads as follows.

Question. Does every discontinuous homomorphism from a unital $C^{*}$-algebra admit a splitting?

When no restrictions are imposed on the domain $C^{*}$-algebra, the answer to this question is "no". We will soon give an example of a discontinuous homomorphism from a liminal separable $\mathrm{C}^{*}$-algebra that does not admit a splitting. Nevertheless, the class of $\mathrm{C}^{*}$-algebras for which a splitting result holds will turn out to be large enough to contain all $\mathrm{AW}^{*}$-algebras and all unital $\mathrm{C}^{*}$-algebras with Hausdorff primitive spectrum.

In order to give as weak as possible a condition for our non-commutative splitting theorem to hold, we require another definition:

Definition 2.2. Let $A$ be a unital $C^{*}$-algebra, and let $X \subset \operatorname{Prim}(A)$. Then $Z(A)$, the center of $A$, is said to separate the points of $X$, if the map

$$
\operatorname{Prim}(A) \rightarrow \operatorname{Prim}(Z(A)), \quad P \mapsto P \cap Z(A)
$$

is injective when restricted to $X$.

Remarks. 1. By [19, Theorem 2.7.5], $\operatorname{Prim}(A)$ is mapped by (1) continuously onto $\operatorname{Prim}(Z(A))$.

2. If $X=\operatorname{Prim}(A)$ (respectively $X$ equals the set of maximal ideals of $A$ ), and $Z(A)$ separates the points of $X, A$ is called central (respectively weakly central) $([\mathbf{1 9}$, Definition 2.7.6]). 
3. All $\mathrm{AW}^{*}$-algebras-and hence all von Neumann algebras-are weakly central ([25, Theorem 2.5]).

4. Assume $A$ to be central. Since $\operatorname{Prim}(A)$ is compact and $\operatorname{Prim}(Z(A))$ is Hausdorff, (1) is a homoemorphism, and $\operatorname{Prim}(A)$ is Hausdorff as well. If conversely $\operatorname{Prim}(A)$ is Hausdorff, then it follows from the Dauns-Hofmann theorem $([17,4.4 .8])$ that $A$ is central.

THEOREM 2.3. Let $A$ be a unital $\mathrm{C}^{*}$-algebra, and let $\theta: A \rightarrow B$ be a homomorphism into a Banach algebra $B$. Suppose that $Z(A)$ separates the points of hull $(\overline{\mathscr{I}(\theta)})$. Then $\theta$ admits a splitting.

Proof. Put $I=\overline{g(\theta)}$, and let $\mathfrak{m}_{1}, \ldots, \mathfrak{m}_{n}$ be the maximal ideals in hull $(I)$. Since $Z(A)$ was assumed to separate the points of hull $(I)$, they give rise to $n$ distinct points $x_{1}, \ldots, x_{n} \in \operatorname{Prim}(Z(A))$. Choose $e_{1}, \ldots, e_{n} \in Z(A)^{+}$, viewed as continuous functions on $\operatorname{Prim}(Z(A))$, such that for $j=1, \ldots, n$

(i) $e_{j} \equiv 1$ on a neighborhood of $x_{j}$, and

(ii) $e_{j} \equiv 0$ on a neighborhood of $\bigcup_{k \neq j}\left\{x_{k}\right\}$.

Define for $j=1, \ldots, n$

$$
\theta_{\text {sing }}^{(j)}(a):=\theta_{\text {sing }}\left(e_{j} a\right) \quad(a \in A) .
$$

The proof that these $\theta_{\text {sing }}^{(j)}$ provide the desired splitting carries over from the commutative case almost verbatim; only Definition $2.1(\mathrm{v})$ requires some more attention. For convenience, we check Definition 2.1(i) and (iv) in detail.

Recall that $\theta_{\text {sing }} \mid I$ is a homomorphism which vanishes on $\mathscr{I}(\theta)$ and hence on $\mathscr{P}(I)$. The element $1-\sum_{j=1}^{n} e_{j}$ is zero on a neighborhood of $\left\{x_{1}, \ldots, x_{n}\right\}$ when viewed as a function on $\operatorname{Prim}(Z(A))$, and hence is contained in $\mathscr{P}(I)$. Thus, we have

$$
\theta_{\text {sing }}(a)-\sum_{j=1}^{n} \theta_{\text {sing }}^{(j)}(a)=\theta_{\text {sing }}\left(\left(1-\sum_{j=1}^{n}\right) a\right)=0 \quad(a \in A),
$$

i.e. $\theta_{\text {sing }}=\sum_{j=1}^{n} \theta_{\text {sing }}^{(j)}$. Now, fix $j$, and let $a, b \in \mathfrak{m}_{j}$. Then $e_{j} a, e_{j} b \in \mathfrak{m}_{j}$, and we have

$$
\theta_{\text {sing }}^{(j)}(a) \theta_{\text {sing }}^{(j)}(b)-\theta_{\text {sing }}^{(j)}(a b)=\theta_{\text {sing }}\left(e_{j} a e_{j} b-e_{j} a b\right)=\theta_{\text {sing }}\left(\left(e_{j}^{2}-e_{j}\right) a b\right)=0,
$$

since $e_{j}^{2}-e_{j}$ vanishes on a neighborhood of $\left\{x_{1}, \ldots, x_{n}\right\}$, and therefore is contained in $\mathscr{P}(I)$.

To see that Definition 2.1(v) holds, let first $a \in \mathscr{P}_{0}\left(\mathfrak{M}_{j}\right)$. Then there is $x \in \mathfrak{u}_{j}$ with $a x=a$. Choose $f_{j} \in Z(A)^{+}$that vanishes on a neighborhood of $\bigcup_{k \neq j}\left\{x_{k}\right\}$, and satisfies $f_{j} e_{j}=e_{j}$. Then $f_{j} x \in I$, and $e_{j} a f_{j} x=f_{j} e_{j} a x=e_{j} a$, i.e. $e_{j} a \in \mathscr{P}_{0}(I)$, which yields $\theta_{\text {sing }}^{(j)}(a)=0$. Now, assume $a \in \mathscr{P}^{+}\left(\mathrm{m}_{i}\right)$. Let $y_{1}, \ldots, y_{m} \in \mathscr{P}_{0}\left(\mathrm{~m}_{j}\right)$ such that $a \leq y_{1}+\ldots+y_{m}$. Then

$$
e_{j} a=e_{j}^{1 / 2} a e_{j}^{1 / 2} \leq e_{j}^{1 / 2} y_{1} e_{j}^{1 / 2}+\ldots+e_{j}^{1 / 2} y_{m} e_{j}^{1 / 2}=e_{j} y_{1}+\ldots+e_{j} y_{m}
$$

Since $e_{i} y_{1}, \ldots, e_{j} y_{m} \in \mathscr{P}_{0}(I)$, we have $e_{j} a \in \mathscr{P}^{+}(I)$ and again $\theta_{\text {sing }}^{(j)}(a)=0$. Hence, $\theta_{\text {sing }}^{(j)}\left(\mathscr{P}\left(\mathrm{m}_{j}\right)\right)=\{0\}$. 
As a consequence of Theorem 2.3, for some classes of non-commutative $\mathrm{C}^{*}$-algebras a perfect analogue of the Bade-Curtis theorem holds.

Corollary 2.4. Let $A$ be a unital $C^{*}$-algebra that is an $\mathrm{AW}^{*}$-algebra or has Hausdorff primitive spectrum; let $B$ be a Banach algebra, and let $\theta: A \rightarrow B$ be $a$ homomorphism with dense range. Then $\theta$ admits a splitting, and $\operatorname{rad}(B)=\Xi(\theta)$.

Proof. As we remarked before, both $\mathrm{AW}^{*}$-algebras and unital $\mathrm{C}^{*}$-algebras with Hausdorff primitive spectrum are weakly central. Since $\overline{\mathscr{I}(\theta)}$ has finite codimension, all primitive ideals in hull $(\overline{\mathscr{Y}(\theta)})$ are maximal ideals, and Theorem 2.3 applies.

To see that $\operatorname{rad}(B)=\Xi(\theta)$, we may assume that $B$ is primitive. We then have to show that $\theta$ is continuous. In view of [11, Theorem 6] and [11, Theorem 11] (see also [24]), it is sufficient to show that $\theta \mid Z(A)$ is continuous. Clearly, $\theta(Z(A)) \subset Z(B)$. Since $B$ is primitive, we have $Z(B) \cong \mathbb{C}$; i.e. $\theta \mid Z(A)$ is a character. Consequently, $\theta \mid Z(A)$ is continuous.

Remarks. 1. A closer inspection of the proof of Theorem 2.3 reveals that the conclusion of the theorem holds as well, if

$$
\mathscr{P}(I \cap J)=\mathscr{P}(I) \cap \mathscr{P}(J)
$$

for all closed ideals $I$ and $J$ of $A$ containing $\overline{\mathscr{Y}(\theta)}$.

2. The following example given in [12] shows that Theorem 2.3 is far from being the best possible result. Let 5 be an infinite-dimensional Hilbert space, and let $x \in \mathscr{B}(5)$ be self-adjoint with $\sigma(x)=[0,1]$. Put $A=\mathrm{C}^{*}\left(x, \mathrm{id}_{\mathfrak{j}}\right)+\mathscr{K}(\mathfrak{5})$. Then $A$ is a $\mathrm{C}^{*}$-algebra, and $A / \mathscr{K}(\tilde{\mathfrak{s}}) \cong C([0,1])$. Under the assumption of the continuum hypothesis, there is a discontinuous homomorphism from $C([0,1])$. It is easy to see that the induced discontinuous homomorphism from $A$ admits a splitting. However, $Z(A)=\mathbb{C i d}_{i}$.

3. It follows from Theorem 2.3 that every homomorphism from a weakly central $\mathrm{C}^{*}$-algebra admits a splitting. In [1], E. Albrecht and H. G. Dales conjectured that if a $C^{*}$-algebra $A$ has only a finite number of $n$-dimensional, irreducible*-representations for every $n \in \mathbb{N}$, then every homomorphism from $A$ into a Banach algebra is continuous. It was pointed out in [24] that if this conjecture were true at least for weakly central $\mathrm{C}^{*}$-algebras, then every homomorphism from a weakly central $\mathrm{C}^{*}$-algebra $A$ which is continuous on $Z(A)$ would be continuous. Consequently, Corollary 2.4 would extend to all homomorphisms from weakly central $C^{*}$-algebras.

3. A counterexample. Let $A$ be a $C^{*}$-algebra, and let $I$ and $J$ be closed ideals of $A$. It is easy to see that $\mathscr{P}(I) \cap \mathscr{P}(J)$ is dense in $I \cap J$, whence

$$
\mathscr{P}(I \cap J) \subset \mathscr{P}(I) \cap \mathscr{P}(J) .
$$

If $A=C(X)$ for a compact Hausdorff space $X$, we clearly have equality in (2). We note in passing that a more general result holds.

Proposition 3.1. Let $A$ be a unital $C^{*}$-algebra, let $I$ and $J$ be closed ideals of $A$, and suppose that $\operatorname{Prim}(A)$ is Hausdorff. Then $\mathscr{P}(I \cap J)=\mathscr{P}(I) \cap \mathscr{P}(J)$.

Proof. Let $a \in \mathscr{P}(I) \cap \mathscr{P}(J)$. By [14, Lemma 5.39], $\{P \in \operatorname{Prim}(I): a \notin P\}$ and $\{P \in \operatorname{Prim}(J): a \notin P\}$ are relatively compact subsets of $\operatorname{Prim}(I)$ and $\operatorname{Prim}(J)$ respectively. 
Hence,

$$
K:=\overline{\{P \in \operatorname{Prim}(A): a \notin P\}}
$$

is a compact subset of $\operatorname{Prim}(A)$ that is disjoint from $\operatorname{hull}(I) \cup \operatorname{hull}(J)=\operatorname{hull}(I \cap J)$. Let $f: \operatorname{Prim}(A) \rightarrow \mathbb{R}^{+}$be a continuous function which is one on $K$ and zero on $\operatorname{hull}(I \cap J)$. By the Daus-Hofmann theorem, there is $x \in Z(A)$ such that

$$
x+P=f(P) 1+P \quad(P \in \operatorname{Prim}(A)) .
$$

Then $a x+P=a+P(P \in \operatorname{Prim}(A))$, and hence $a x=a$. On the other hand, $x$ is clearly contained in $I \cap J$, which yields $a \in \mathscr{P}(I \cap J)$.

The following example, which appears (somewhat disguised) already in [16], was communicated by G. K. Pedersen. It shows that already for rather simple $\mathrm{C}^{*}$-algebras the inclusion (2) can be proper, even if both $I$ and $J$ are assumed to be primitive ideals of codimension one. Moreover, the $\mathrm{C}^{*}$-algebra in this example will turn out to be the domain of a homomorphism which does not admit a splitting.

ExAmple. Let $\delta \in(0,1)$, and let $A$ be the $C^{*}$-algebra of all continuous functions $f:[0, \delta] \rightarrow M_{2}$, where $M_{2}$ is the algebra of all $2 \times 2$-matrices with complex entries, such that $f(0)$ is a diagonal matrix. The irreducible *-representations of $A$ are either point evaluations at $t \in(0, \delta]$ or the maps

$$
A \ni f=\left[\begin{array}{ll}
f_{11} & f_{12} \\
f_{21} & f_{22}
\end{array}\right] \mapsto f_{i j}(0) \quad(j=1,2),
$$

which yields in particular that $A$ is liminal. Let $\mathrm{m}_{1}$ and $\mathrm{m}_{2}$ denote the respective kernels of the maps (3). Consider the functions $p, q:[0, \delta] \rightarrow M_{2}$, where

$$
p(t)=\left[\begin{array}{ll}
0 & 0 \\
0 & 1
\end{array}\right] \text { and } q(t)=\left[\begin{array}{cc}
t & \sqrt{t-t^{2}} \\
\sqrt{t-t^{2}} & 1-t
\end{array}\right] \quad(t \in(0, \delta])
$$

Then $p$ and $q$ are projections contained in $\mathfrak{m}_{1}$. It is easy to see that $\rho\left(C^{*}(p, q)\right)=\rho\left(\mathrm{m}_{1}\right)$ for every irreducible ${ }^{*}$-representation $\rho$ of $A$, which by $[5,11.1 .6]$ implies $C^{*}(p, q)=m_{1}$. By $[17,5.6 .2]$ and $[17,5.6 .3], C^{*}(p, q) \subset \mathscr{P}\left(m_{1}\right)$ holds; i.e. we have $\mathscr{P}\left(m_{1}\right)=m_{1}$. An analoguous reasoning where $p$ and $q$ are replaced by $1-p$ and $1-q$ shows that $\mathscr{P}\left(\mathfrak{m}_{2}\right)=\mathfrak{m}_{2}$ as well. However, $\mathscr{P}\left(\mathfrak{m}_{1} \cap \mathfrak{m}_{2}\right)$ is the ideal of all functions in $A$ that vanish on a neighborhood of 0 . Hence

$$
\mathscr{P}\left(\mathfrak{m}_{1} \cap \mathfrak{m}_{2}\right) \subseteq \mathscr{P}\left(\mathfrak{m}_{1}\right) \cap \mathscr{P}\left(\mathfrak{m}_{2}\right) .
$$

To construct a homomorphism from $A$ that does not admit a splitting, note that $A$ can be viewed as a subalgebra of $M_{2}(C([0, \delta]))$, the algebra of all $2 \times 2$-matrices with entries in $C([0, \delta])$ :

$$
A \cong\left\{\left[\begin{array}{ll}
f_{11} & f_{12} \\
f_{21} & f_{22}
\end{array}\right] \in M_{2}(C([0, \delta])): f_{12}, f_{21} \in \mathfrak{m}_{0}\right\},
$$

where $\mathfrak{m}_{0}$ is the maximal ideal of $C([0, \delta])$ consisting of all functions that vanish at 0 . Let $\mathfrak{p}$ be a non-maximal prime ideal of $C([0, \delta])$ contained in $m_{0}$. Since $C([0, \delta])$ is separable, $C([0, \delta]) / \mathfrak{p}$ has cardinality $2{ }^{\wedge}$. Assuming the continuum hypothesis, there is a discontinuous homomorphism $\theta$ from $C([0, \delta])$ with $\mathfrak{p}$ as its kernel into a Banach algebra $B . \theta$ 
induces a homomorphism $M_{2}(C([0, \delta])) \rightarrow M_{2}(B)$. Let its restriction to $A$ be denoted by $\Theta$. Obviously,

$$
\overline{\mathscr{I}(\Theta)}=\left\{\left[\begin{array}{ll}
f_{11} & f_{12} \\
f_{21} & f_{22}
\end{array}\right] \in A: f_{11}, f_{22} \in \mathrm{m}_{10}\right\},
$$

i.e. hull $\overline{(\Phi(\Theta)})=\left\{m_{1}, m_{2}\right\}$. If $\Theta$ admitted a splitting, then-by Definition $2.1(v)$ and the fact that $\mathscr{P}\left(\mathrm{nm}_{j}\right)=\mathrm{mt}_{j}-\Theta_{\text {sing }}^{(j)}\left(\mathrm{mi}_{j}\right)=\{0\}(j=1,2)$, and hence $\Theta_{\text {sing }}(\overline{\mathscr{F}(\Theta)})=\{0\}$. It would follow that $\Theta$ is continuous. This contradicts the definition of $\Theta$.

4. Another non-commutative splitting result. In $[10], B$. E. Johnson further refined the structural analysis of discontinuous homomorphisms from $C(X)$, making use of the Stone-Čech compactification. We seek non-commutative extensions of Johnson's results ([10, Theorem 2.5]).

We start with a technical lemma.

Lemma 4.1. Let $A$ be a $C^{*}$-algebra, let $I \subset A$ be a closed ideal, and let $\theta: I \rightarrow B$ be a homomorphism into a Banach algebra $B$. Define

$$
\mathscr{J}(\theta):=\{a \in A: \operatorname{IaI} \subset \mathscr{I}(\theta)\} .
$$

Then $\mathscr{g}(\theta)$ is an ideal of $A$ whose closure has finite codimension, and the restriction of $\theta$ to $\mathscr{P}(\overline{\mathscr{g}(\theta)}) \cap I$ is continuous.

Proof. Put $J:=\overline{\mathscr{J}(\theta)}$. By [4, Theorem 4.4.13], $J$ has finite codimension. Let $x \in \mathscr{P}(J) \cap I$, and assume first that $x \geq 0$. Then, by [17, 5.6.2], $x^{1 / 3} \in \mathscr{P}(J) \cap I$ as well; in particular, $x^{1 / 3} \in \mathscr{I}(\theta) \cap I$. By the definition of $\mathscr{F}(\theta), x=x^{1 / 3} x^{1 / 3} x^{1 / 3} \in \mathscr{I}(\theta)$. By Proposition 1.3, $\theta \mid \mathscr{I}(\theta)$ is continuous. Let $\gamma:=\|\theta \mid \mathscr{I}(\theta)\|$. Then we have $\|\theta(x)\| \leq \gamma\|x\|$. If $x$ is an arbitrary element of $\mathscr{P}(J) \cap I$, let $x_{1}, x_{2}, x_{3}, x_{4}$ be positive elements in $\mathscr{P}(J) \cap I$ such that

(i) $\left\|x_{j}\right\| \leq\|x\| \quad(j=1,2,3,4)$,

(ii) $x=\left(x_{1}-x_{2}\right)+i\left(x_{3}-x_{4}\right)$.

(By $[17,5.6 .2]$, the standard decomposition of $x$ will do.) Then

$$
\|\theta(x)\| \leq \gamma\left\|x_{1}\right\|+\ldots+\gamma\left\|x_{4}\right\| \leq 4 \gamma\|x\|,
$$

which yields that $\theta \mid \mathscr{P}(J) \cap I$ is continuous.

For a $\mathrm{C}^{*}$-algebra $A$ let $\mathcal{M}(A)$ denote its multiplier algebra (see [17] for a definition and basic properties). If $X$ is a locally compact Hausdorff space, then $\mathcal{M}\left(C_{0}(X)\right) \cong C_{b}(X)$, the algebra of continuous bounded functions on $X$, whose primitive spectrum is the Stone-Cech compactification of $X$. Therefore, the multiplier algebra of a $\mathrm{C}^{*}$-algebra can be viewed as a non-commutative analogue of the Stone-Čech compactification. For more on this aspect of multiplier algebras see the survey article [18].

Theorem 4.2. Let $A$ be a non-unital $C^{*}$-algebra, and let $\theta: A \rightarrow B$ be a non-zero homomorphism into a Banach algebra $B$ such that $\theta(\mathscr{P}(A))=\{0\}$.

(i) There is a proper closed ideal $J$ of $\mathcal{M}(A)$ that contains $A$ and has finite codimension in $\mathcal{M}(A)$ such that $\theta(\mathscr{P}(J) \cap A)=\{0\}$. 
(ii) Moreover, assume that $Z(\mathcal{M}(A))$ separates the points of $\operatorname{hull}(J)$ in $\operatorname{Prim}(\mathcal{M}(A))$, say $\mathfrak{m}_{1}, \ldots, \mathfrak{m}_{n}$. Then there are homomorphisms $\theta_{1}, \ldots, \theta_{n}: A \rightarrow B$ such that

(a) $\theta=\theta_{1}+\ldots+\theta_{n}$,

(b) $\theta_{j}\left(A \cap \mathscr{P}\left(\mathrm{m}_{j}\right)\right)=\{0\} \quad(j=1, \ldots, n)$,

(c) $\operatorname{ker} \theta=\bigcap_{j=1}^{n} \operatorname{ker} \theta_{j}$.

Proof. To show (i), view $A$ as a closed ideal of $\mathcal{M}(A)$, and define $J=\overline{\mathscr{J}(\theta)}$, as in Lemma 4.1. Then $J$ has finite codimension in $\mathcal{M}(A)$, and $\theta \mid \mathscr{P}(J) \cap A$ is continuous. Since $\mathscr{J}(\theta) \cap A \supset \mathscr{I}(\theta) \supset \mathscr{P}(A), J \cap A \supset A$; i.e. $J$ contains $A$. So, $\mathscr{P}(J) \cap A$ is a dense ideal in $A=J \cap A$, and therefore contains $\mathscr{P}(A)$. Since by assumption $\theta$ vanishes on $\mathscr{P}(A)$, we have $\theta(\mathscr{P}(J) \cap A)=\{0\}$.

The proof of (ii) is quite similar to the proof of Theorem 2.3. We choose elements $e_{1}, \ldots, e_{n} \in Z(A)^{+}$. such that each $e_{j} \equiv 1$ on a neighborhood of $x_{j}$, where $x_{j}$ is the point in the spectrum of $Z(A)$ corresponding to $\mathrm{m}_{j}$, and $e_{j} \equiv 0$ on a neighborhood of $\bigcup_{k \neq j}\left\{x_{k}\right\}$. Moreover, we can assume that $e_{j} e_{k}=0(j \neq k)$. Then define for $j=1, \ldots, n$

$$
\theta_{j}(a):=\theta\left(e_{j} a\right) \quad(a \in A) .
$$

Emulating the proof of Theorem 2.3 , we see easily that $\theta_{1}, \ldots, \theta_{n}$ are homomorphisms, and that (a) and (b) hold. Since for all $a \in A, \theta_{j}(a) \theta_{k}(a)=0(j \neq k)$, we have

$$
\theta_{j}(a) \theta(a)=\theta_{j}(a)^{2} \quad(a \in A, j=1, \ldots, n),
$$

which implies (c).

Theorem 4.3. Let $A$ be a unital $C^{*}$-algebra, and let $\theta: A \rightarrow B$ be a discontinuous homomorphism into a Banach algebra $B$. Then there is a proper closed ideal I of $A$ with finite codimension in $A$, and a proper closed ideal $J$ of $\mathcal{M}(I)$ that contains $I$ and has finite codimension in $\mathcal{M}(I)$ such that $\mathscr{P}(J) \cap I \subset \mathscr{I}(\theta)$.

Proof. Put $I=\overline{\mathscr{I}(\theta)}$, and apply Theorem $4.2(\mathrm{i})$ to $\theta_{\text {sing }}: I \rightarrow B$. Then $\theta_{\text {sing }}(\mathscr{P}(J) \cap I)$ $=\{0\}$; i.e. $\theta\left|\mathscr{P}(J) \cap I=\theta_{\text {cont }}\right| \mathscr{P}(J) \cap I$. This yields that $\theta \mid \mathscr{P}(J) \cap I$ is continuous, and hence, by Proposition 1.3, that $\mathscr{P}(J) \cap I \subset \mathscr{I}(\theta)$.

\section{REFERENCES}

1. E. Albrecht and H. G. Dales, Continuity of homomorphisms from $\mathrm{C}^{*}$-algebras and other Banach algebras, in J. M. Bachar, W. G. Bade, P. C. Curtis Jr., H. G. Dales, and M. P. Thomas, Editors, Radical Banach algebras and automatic continuity, Volume 975 of Lecture Notes in Mathematics, (Springer Verlag, 1983), 375-396.

2. W. G. Bade and P. C. Curtis Jr, Homomorphisms of commutative Banach algebras, Amer. J. Math. 82 (1960), 589-608. $647-734$.

3. H. G. Dales, A discontinuous homomorphism from $C(X)$. Amer. J. Math. 101 (1979),

4. H. G. Dales, Banach algebras and automatic continuity (Oxford University Press, in preparation).

5. J. Dixmier, $C^{*}$-algebras, (North-Holland Publishing Company, 1977).

6. J. Esterle, Solution d'un problème d'Erdös, Gillman et Henriksen et application à l'étude des homomorphismes de $C(K)$, Acta Math. Acad. Sci. Hungar. 30 (1977), 113-127. 
7. J. Esterle, Injection des semigroupes divisibles dans les algèbres de convolution et construction d'homomorphismes discontinus de $C(K)$, Proc. London Math. Soc. (3) 36 (1978), $59-85$.

8. J. Esterle, Sur l'existence d'un homomorphisme discontinu de $C(K)$, Proc. London Math. Soc. (3) 36 (1978), 46-58.

9. B. E. Johnson, Continuity of homomorphisms of algebras of operators, II, J. London Math. Soc. (2) 1 (1969), 81-84. $37-58$.

10. B. E. Johnson, Norming $C(\Omega)$ and related algebras, Trans. Amer. Math. Soc. 220 (1974),

11. K. B. Laursen, Central factorization in $\mathrm{C}^{*}$-algebras and continuity of homomorphisms, $J$. London Math. Soc. (2) 28 (1983), 123-130.

12. K. B. Laursen, Central factorization in $\mathrm{C}^{*}$-algebras and its use in automatic continuity, in F. Greenleaf and D. Gulick, editors, Banach algebras and several complex variables, Contemporary Mathematics, 32 (1984), 169-176.

13. K. B. Laursen and A. M. Sinclair, Lifting matrix units in $\mathrm{C}^{*}$-algebras, II, Math. Scand. 37 (1975), 167-172. (1976)

14. A. J. Lazar and D. C. Taylor, Multipliers of Pedersen's ideal, Mem. Amer. Math. Soc. 169

15. G. K. Pedersen, Measure theory for $C^{*}$-algebras, Math. Scand. 19 (1966), 131-146.

16. G. K. Pedersen. Measure theory for $C^{*}$-algebras, II, Math. Scand. 22 (1968), 63-74.

17. G. K. Pedersen, $C^{*}$-algebras and their automorphism groups (Academic Press, 1979).

18. G. K. Pedersen, The corona construction, in J. B. Conway and B. B. Morrel, editors, Operator Theory: Proceedings of the 1988 GPOTS-Wabash Conference, Pitman Research Notes in Mathematics Series, 225 (1990), 49-92.

19. C. E. Rickart, General theory of Banach algebras (Van Nostrand, 1960).

20, A. M. Sinclair, Homomorphisms from C*-algebras, Proc. London Math. Soc. (3) 29 (1974), 435-452. $165-174$.

21. A. M. Sinclair, Homomorphisms from $C_{0}(\mathbb{R}), J$. London Math. Soc. (2) 11 (1975),

22. A. M. Sinclair, Automatic continuity of linear operators, London Mathematical Society Lecture Notes Series (Cambridge University Press, 1976).

23. A. M. Sinclair, Corrigendum: Homomorphisms from $\mathrm{C}^{*}$-algebras, Proc. London Math. Soc. (3) 32 (1976), 322.

24. D. W. B. Somerset, Discontinuous homomorphisms from $\mathrm{C}^{*}$-algebras, Math. Proc. Cambridge Philos. Soc. 110 (1991), 147-150.

25. F. B. Wright, A reduction for algebras of finite type, Ann. of Math. 60 (1954), 560-570.

\author{
Department of Mathematics \\ UNIVERSITY OF CALIFORNIA \\ BERKELEY, CA 94720 \\ U.S.A. \\ vrunde@math . berkeley .edu \\ Present address: \\ Facubereich 9 Mathematik \\ UNIVERSITÄT DES SAARLANDES \\ POSTFACH 151150 \\ 66041 SAARBRÜCKEN \\ Germany
}

